\title{
Fringe pattern denoising by image dimensionality reduction
}

\author{
J. Vargas ${ }^{\mathrm{a}, *}$, C.O.S. Sorzano ${ }^{\mathrm{a}}$, J. Antonio Quiroga ${ }^{\mathrm{b}}$, J.C. Estrada ${ }^{\mathrm{c}}$, J.M. Carazo ${ }^{\mathrm{a}}$ \\ a Biocomputing Unit, Centro Nacional de Biotecnología-CSIC, C/Darwin 3, 28049 Cantoblanco (Madrid), Spain \\ b Optics Department, Universidad Complutense de Madrid, Facultad de CC. Físicas, Ciudad Universitaria s/n, 28040 Madrid, Spain \\ ' Centro de Investigaciones en Óptica A. C., Loma del Bosque 115, Col. Lomas del Campestre, 37150 León (Guanajuato), Mexico
}

\section{A R T I C L E I N F O}

\section{Article history:}

Received 7 December 2012

Received in revised form

28 January 2013

Accepted 20 February 2013

Available online 16 March 2013

Keywords:

Interferometry

Fringe processing

Denoising

Dimensionality reduction

\begin{abstract}
A B S T R A C T
Noise is a key problem in fringe pattern processing, especially in single frame demodulation of interferograms. In this work, we propose to filter the pattern noise using a straightforward, fast and easy to implement denoising method, which is based on a dimensionality reduction approach, in the sense of image rank reduction. The proposed technique has been applied to simulated and experimental ESPI interferograms obtaining satisfactory results.
\end{abstract}

(c) 2013 Elsevier Ltd. All rights reserved.

\section{Introduction}

Denoising of fringe patterns is an important preprocessing step, especially in single frame demodulation problems [1], where the modulating phase has to be extracted from a single interferogram or in Electronic Speckle Pattern Interferometry (ESPI) [1], where the strong grain-shaped random noise of ESPI patterns leads to heavy restrain in phase value extraction. Without noise, the fringe pattern processing becomes much easier and straightforward. Typically, the first attempt in order to denoise a fringe pattern consists in filtering using an average convolution kernel (typically by a Gaussian kernel) one or several times in an iterative way. However, such a denoising process will cause a hopeless bluring effect in the interferogram fringes and then, a fringe contrast reduction in the fringe pattern. Recently, in order to solve this problem different methods based on partial differential equations (PDEs) have been proposed.

The filtering method based on partial differential equations (PDEs) has become an interesting research topic [2-4]. A great deal of the success of PDEs applications in image processing can be attributed to their basic characteristics: local and high frequency features preserving. All noise filtering methods based on PDEs have a similar theoretical foundation that is the diffusion process or heat equation,

$\frac{\partial I}{\partial t}=c \Delta I, \quad I\left(t_{0}\right)=I, \quad c>0 \in R$

\footnotetext{
* Corresponding author. Tel.: +34 62 0486940; fax: + 34915854506 .

E-mail addresses: jvargas@cnb.csic.es, jvargas@fis.ucm.es (J. Vargas).
}

In Eq. (1), $I$ is the image to process, $\Delta$ is the Laplace operator, $t$ is the temporal magnitude used as the iterating parameter and $c$ is the diffusion coefficient that is a positive valued scalar. The diffusion process as shown in expression (1) is equivalent to a smoothing process with a Gaussian kernel [2]. If $c$ equals to 1 , solving Eq. (1) is analogous to convolving the input image with a Gaussian kernel with a standard deviation of $\sigma=\sqrt{2 T}$, with $T$ the total time interval that corresponds to the total number of iterations [2]. Filtering using a Gaussian kernel is not, in general, the best choice as important image features will be smoothed and blurred. Perona and Mallik [2] addressed this issue modifying expression (1) and constructing a nonlinear adaptive denoising process, where diffusion takes place using a spatially variable diffusion coefficient in order to reduce the smoothing effect near edges. However, the diffusion scheme proposed in [2] is isotropic as locally the diffusion process has no favorite directions [4]. In highly oriented structures as fringe patterns, it is more appropriate to transform this isotropic process into an anisotropic one. A convenient anisotropic scheme can be found using a diffusion tensor instead of the diffusion scalar [3-4]. In [3] it is presented a method that filters only along fringe orientation. In Ref. [4] it is shown an approach that improves [3] diffusing along and across fringe orientation using an adaptive method based on a fringe density estimation. Note that both methods consist in an iterative denoising process that uses a rectangular average convolution window. The shape of this averaging convolution window is different in Tang [3] and Wang [4] methods. This window is adaptively oriented perpendicularly to the fringe orientation so the diffusion is mainly along and non across the fringes, avoiding therefore a fringe contrast reduction that will appear in the 
interferogram, if we use a typical Gaussian kernel. The Wang method [4] is very effective in noise suppression but presents some degree of image artifacts, especially if a low number of iterations are employed. Other drawbacks are the computational cost and processing time that this method demands; in our experience it needs around 200 iterations to effectively denoise an ESPI pattern. Additionally, this method requires a large number of user defined parameters. In our implementation, we need six parameters [5], that typically are difficult to be tuned to the problem at hand.

Dimensional reduction approaches consist in statistical procedures that aim to transform the original data set, defined in a high dimensional space, to another space of fewer dimensions subtracting the redundant or irrelevant data without loss of valuable information [7]. In many areas of artificial intelligence, information retrieval and data mining, one is confronted with intrinsically low dimensional data lying in a very high dimensional space [6-9]. Exemplary cases are biological sciences, where hundred or thousand of data can be reported by a single experiment and data mining techniques are needed to separate relevant from unimportant information. We can consider that an image of size $N \times M$ "lives" in a vector subspace of dimension $N \times M$. In this context, image dimensionality refers to the degrees of freedom or possible independent pixel values that an image can take. Interferograms follow a mathematical model in which pixel values can be correlated far away from their location and therefore, the pixel values are no longer independent and therefore the number of degrees of freedom is strongly reduced.

In this work, we propose to use a straightforward denoising method, fast and easy to implement based on a dimensionality reduction method [6-9], in the sense of image rank reduction and that uses the Singular Value Decomposition algorithm (SVD). The paper is organized as follows. In the next section, we present the theoretical basis of our method. Then, we compare our technique with Tang [3] and Wang [4] methods, as well as, with a typical convolution-based denoising filter. Afterwards, we test our proposed denoising method with real ESPI patterns, and finally, conclusions are drawn.

\section{Theoretical foundations}

The simplest model for an interferogram is given by,

$I(x, y)=I_{a}(x, y)+I_{b}(x, y) \cos (\Phi(x, y))$

where $I(x, y)$ is the intensity at pixel $(x, y), I_{a}$ and $I_{b}$ are the background and modulation terms and $\Phi$ is the modulating phase. Moreover, in interferometry, $I_{a}, I_{b}$ and $\Phi$ are typically smooth functions and therefore, in general, the dimensionality of an interferogram as shown in expression (1) is low. A convenient image dimensionality descriptor is the image rank, that it is defined as the number of independent image rows or columns. Obviously, high or low image rank implies high or low image dimensionality.

Fringe patterns obtained in experimental setups are always affected by noise. Typically, this noise is Gaussian and additive so expression (1) has to be rewritten to,

$I(x, y)=I_{a}(x, y)+I_{b}(x, y) \cos (\Phi(x, y))+\eta(x, y)$

where $\eta$ is the noise term characterized by a standard deviation of $\sigma$. In this case, the dimensionality of the image and therefore the image rank is increased with respect to the noiseless interferogram shown in Eq. (2).

The proposed denoising algorithm is based on decomposing the noisy fringe pattern into a sum of component signals, in such a way that these component signals are sorted taking into account how representative they are in the composed image. If one of these component signals is blocked, the reconstructed image by the remaining signals will have smaller dimensionality and rank than the original one. In our proposed method we have used the Singular Value Decomposition (SVD) factorization method to perform the dimensionality reduction. The SVD algorithm consists in a unique factorization of a matrix of the form,

$I=U D V^{T}$

where $I$ is a real image and has dimension $N \times M$ with $M \geq N, U$ and $V^{T}$ are $N \times N$ and $M \times M$ orthogonal matrixes and $D$ is a $N \times M$ diagonal matrix. The columns of $U$ and $V$ will be denoted respectively as, $u_{k}, v_{k}$, and are called left and right singular vectors of $I$. The entries of $D$ are only non-zero on the diagonal and are known as the singular values. The left and right singular vectors are eigenvectors of $I \times I^{T}$ and $I^{T} \times I$ respectively, and the singular values, $\lambda_{k}$ are real and positive valued. The rank, and therefore the dimensionality of $I$, is directly related to the $D$ matrix. The rank of $I$, that we will denote as $r$, is the number of non-zero entries in $D$. The image or fringe pattern can be rewritten taking into account the SVD factorization as,

$I=\sum_{k=1}^{r} u_{k} \lambda_{k} v_{k}^{T}$

From expression (5) we can see that the image $I$ can be decomposed in terms of a summation of signals as,

$I=\sum_{k=1}^{r} I_{k}$

where the different signals $I_{k}$ are characterized by its singular value $\lambda_{k}$ and they are orthogonal so,

$\left\langle I_{i}, I_{j}\right\rangle=\sum_{x, y} I_{i} I_{j}=0$

with $i$ and $j$ integer values, $i \neq j$ and $i, j \leq r$. The largest singular value, that by construction has lowest $k$ index and that corresponds to one, represents the most significant mode of variation of the data; the second component, that has $k$ equals to two, corresponds to the following largest singular value and most significant mode and so on. This important result means that if we truncate the SVD matrix factorization imposing that all singular values smaller than the $n$th singular value, with $n<r$, are zero, we obtain the closest $n$-rank matrix to $I$. This truncated representation of $I$ matrix is given by,

$\tilde{I}^{n}=\Theta^{n}[I]=\sum_{k=1}^{n} \mathbf{u}_{k} \lambda_{k} \mathbf{v}_{k}^{T}$

where $\Theta^{n}$ denotes the $n$-dimensional reduction operator and $n$ is the number of largest singular values that are not truncated and will be used in the image reconstruction by Eq. (8). Note that in a practical point of view, the $\Theta^{n}$ operator consists in set to zero the smallest $r-n$ singular values, denoted as $\lambda_{k}$ in Eq. (5) and with $k>n$. Therefore, the number of singular values that will be set to zero corresponds to $r-n$. The term closest in this context means that $\tilde{I}^{n}$ minimizes the sum of squares of the difference between $\tilde{I}^{n}$ and $I$. Therefore, $\tilde{I}^{n}$ corresponds to the "closest" $n$-rank image approximation of $I$.

Suppose that we have a fringe pattern formed by perfectly parallel vertical fringes. In this restricted case, any image row can be considered as a copy of the first row and then, the image rank is exactly one. Therefore, in this case, all elements of $D$ will be zero except the first one, that is $\lambda_{1}$. Now suppose that this fringe pattern is affected by noise. The rank of this image is not anymore one and the relative value between the first and second singular values depends on the image Signal to Noise Ratio (SNR). In this case, we can efficiently subtract the noise truncating the rank of the image in order to obtain the "closest" 1-rank fringe pattern. The noisy 
fringe pattern mentioned above can be mathematically represented as,

$I(x, y)=\cos (\omega x)+\eta(x, y)$

where $\omega$ is a positive real number and $I$ is an image of size $N \times M$. The eigenvalues of $I$, that are $\lambda$, can be obtained from the eigenvalues of $I I^{T}$ as,

$I \times I^{T}=\left(U D V^{T}\right)\left(V D^{T} U^{T}\right)=U F U^{T}$

where $F=D D^{T}$. Note that $V$ is an orthogonal matrix and, therefore $V V^{T}$ equals to the identity matrix. The eigenvalues of $I \times I^{T}$ corresponds to $\lambda_{i}^{2}$, for $i \in[1, r]$. Taking into account expression (9) we have that,

$I(x, y) I(x, y)^{T}=A+B(x, y)$

where $A$ and $B(x, y)$ are $N \times N$ matrixes. The elements of $A$ matrix have all the same value that corresponds to $\sum_{x=1}^{M} \cos (\omega x)^{2}$. The elements of $B$ matrix are equal to,

$B(x, y)=\left(\cos (\omega x) \eta(x, y)+\eta(x, y)^{T} \cos (\omega x)+\eta(x, y) \eta(x, y)^{T}\right) . \quad$ Note that if the image $I$ is not affected by noise, we have that $\eta(x, y)=0$ and then, $I \times I^{T}=A$, as $B$ matrix is zero. In this noiseless case, the image $I$ has only one eigenvalue $\lambda_{1}$ as the rank of $A$ matrix is one. This matrix has only one eigenvalue different to zero and equals to $\lambda_{1}^{2}=\sum_{x=1}^{M} \sum_{y=1}^{N} \cos (\omega x)^{2}$. On the other hand, if $\eta(x, y) \neq 0$, then it will appear $r$ different eigenvalues, with $r$ the image rank.

In Fig. 1(a) and (b), we show a simulated fringe pattern formed by perfectly parallel fringes without and with noise as shown in (9) respectively. Fig. 1(c) and (d) shows respective plots of the diagonal of the recovered $D$ matrix along $k$ index for the pattern without and with noise. As can be seen from Fig. 1(c) and (d), the fringe pattern without noise only presents one non-zero eigenvalue for $k$ equals to one that corresponds to $\lambda_{1}=353$ in arbitrary units (a.u). This value matches exactly with the value obtained numerically by
$\lambda_{1}^{2}=\sum_{x=1}^{M} \sum_{y=1}^{N} \cos (\omega x)^{2}$. The noisy pattern has a large set of non-zero eigenvalues, with decreasing values along increasing values of $k$ index. In this case, $\lambda_{1}$ corresponds to 356 (a.u). Finally, Fig. 1(e) and (f) shows the reconstructed fringe patterns $\tilde{I}^{n}$ with $n=1$, for the patterns without and with noise. From Fig. 1(f) we see that this dimensionality reduction method can efficiently subtracts the noise presented in Fig. 1(b), but this reconstructed fringe pattern presents image artifacts parallel to the image $x$ and $y$ axis. An efficient way to subtract these oriented image artifacts consists in performing dimensionality reduction over a set of rotated versions of the fringe pattern. These rotated versions of $I$ will be denoted as $I_{\theta}=R_{\theta}(I)$, where $R_{\theta}$ is the image rotating operator and $\theta$ is the rotating angle. The rotation operator performs a geometric transform which maps each element in the input image $I$ onto a new position in the output image by rotating it through an angle $\theta$ about the image center. The dimensionality reduced fringe patterns will be denoted as $\tilde{I}_{\theta}^{n}$ and corresponds to,

$\tilde{I}_{\theta}^{n}=R_{\theta}^{-1}\left(\Theta^{n}\left[R_{\theta}(I)\right]\right)$

In order to keep the entire image after the rotation, we perform a zero padding process and we rotate this extended image. Note that the dimensionality reduced fringe patterns $\left(\tilde{I}_{\theta}^{n}\right)$, given in Eq. (12) have the same size than the original image $I$ because the additional zeros are subtracted. For each angle $\theta$, we also obtain the sum of the non-blocked $n$ singular values, that we will denote as $\varepsilon_{\theta}=\sum_{i=1}^{n}\left(D_{\theta}\right)_{i}$, where $D_{\theta}$ is the diagonal matrix for $R_{\theta}(I)$ image. We perform $K$ pattern rotations with $K=2 \pi / \theta$. Finally, we obtain the denoised fringe pattern as,

$\tilde{I}=\sum_{\theta} \tilde{I}_{\theta}^{n} \varepsilon_{\theta} / \sum_{\theta} \varepsilon_{\theta}$

Note that each pattern $\tilde{I}_{\theta}^{n}$ has image artifacts with different favorite orientations defined by the rotation angle $\theta$. The combination of $\tilde{I}_{\theta}^{n}$ for different rotating angles, suppress these oriented artifacts. As shown, the proposed denoising method only requires two user a

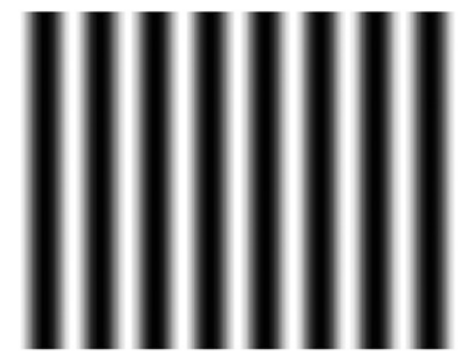

b

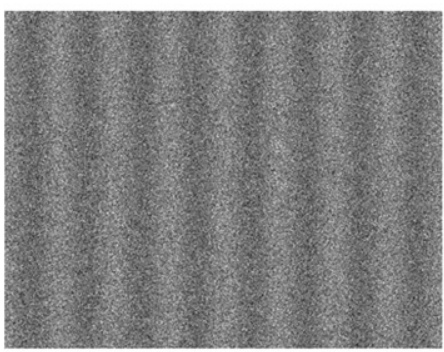

C

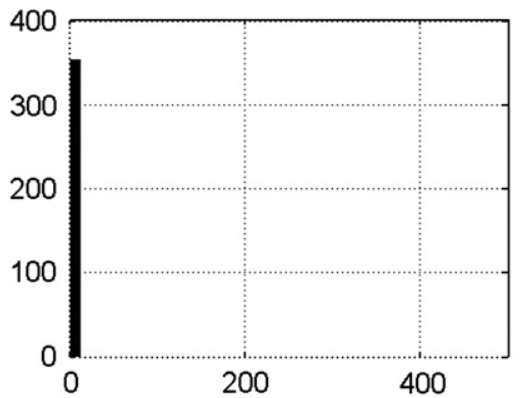

d

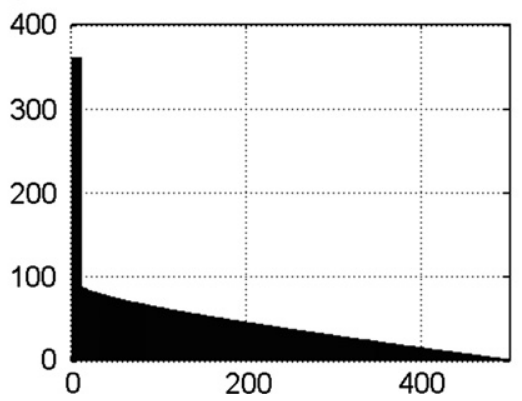

e

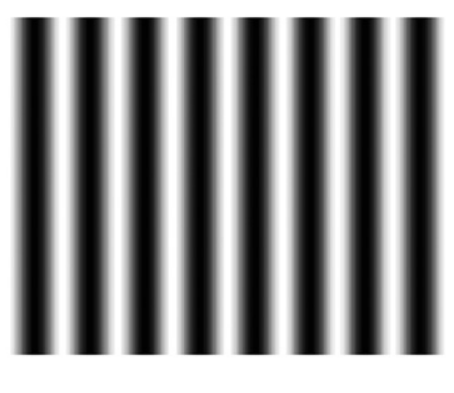

f

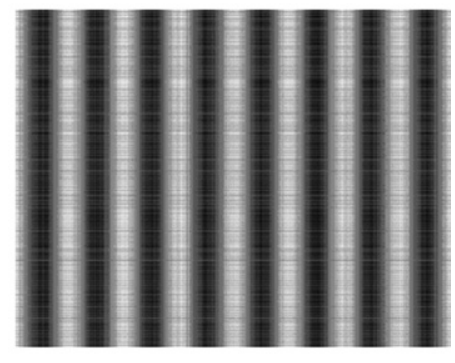

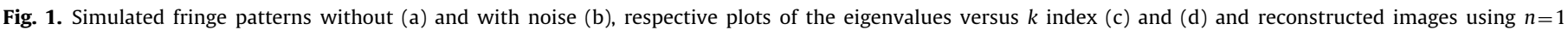
(e) and (f). 
defined parameters, the number of rotations $K$ and the number of eigenvalues to keep $n$. Observe, that the proposed dimensionality reduction method can be performed in an iterative way if desired. We can use the output image of the algorithm as the input image of an additional denoising process by the proposed dimensionality reduction method. In this sense, we can define the number of iterations of the denoising method as the number of times we perform the proposed dimensionality reduction approach.

The proposed method is based on assuming that the background, modulation and phase terms are smooth signals. In this case, the resultant interferogram pixel values can be correlated far away from their location and therefore, the pixel values are no longer independent. In this case, the number of degrees of freedom can be strongly reduced without losing important information. Note that in interferograms with highly locally varying background, modulation and/ or phase signals, the applicability of the proposed method can be limited.

\section{Numerical analysis}

We have tested the proposed dimensionality reduction method (DR) using a simulation. We compare the results obtained by the DR approach with the results obtained by the Tang [3] and Wang [4] methods and also by a typical convolution-based denoising filter. We use a simulated fringe pattern affected by additive Gaussian noise with zero mean and standard deviation of one. The mathematical expressions of the simulated fringe pattern shown in Fig. 2(a) corresponds to,

$I(x, y)=\cos \left(1.5 P(x, y)+x^{2}+y^{2}\right)+N(x, y)$

where $P(x, y)$ is the Matlab built in function peaks, $N(x, y)$ is the added noise that has a standard normal distribution with zero mean and $x, y$ correspond to pixel coordinates with the origin of coordinates placed in the image center. The size of the image is $350 \times 350$. The Signal to Noise ratio (SNR) is of $25 \%$. In Fig. 2(b)-(f), we show the resultant denoised patterns when we use the proposed approach, the Wang and Tang methods and a convolution-based denoising filter with a kernel of size $3 \times 3$ after 1 (Fig. 2(e)) and 20 iterations (Fig. 2(f)). We use 15 rotations $(K=15)$ and a rank truncation of $25(n=25)$ as input arguments of the DR method and we perform two iterations. The results obtained by the Tang and Wang approaches are acquired using the following parameters. The diffusion speed parallel $\left(\alpha_{2}\right)$ and perpendicular $\left(\alpha_{1}\right)$ to the fringe orientation are $\alpha_{2}=1$ at every pixel, while $\alpha_{1}$ depends on the local fringe density map, following the Wang method. Note that the Tang method establishes that $\alpha_{1}=0$ at each pixel location. Additionally, $\sigma$ and $\rho$ that are space regularization parameters corresponding to the a

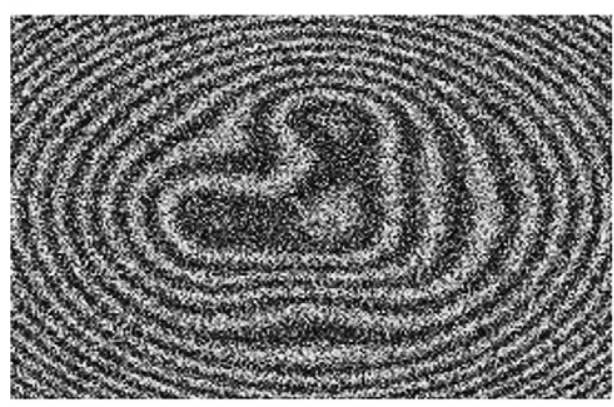

C

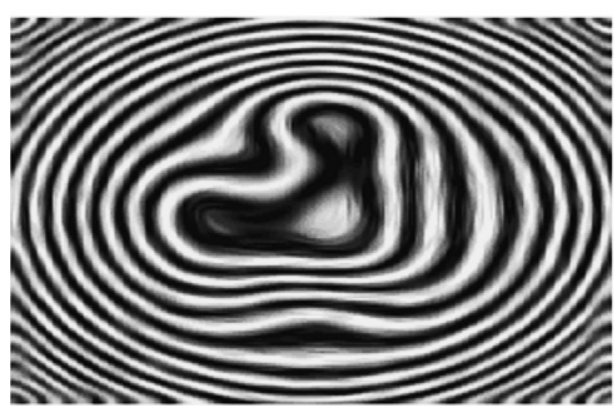

e

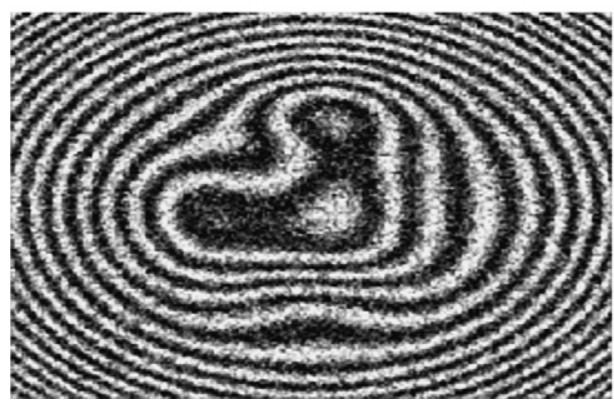

b

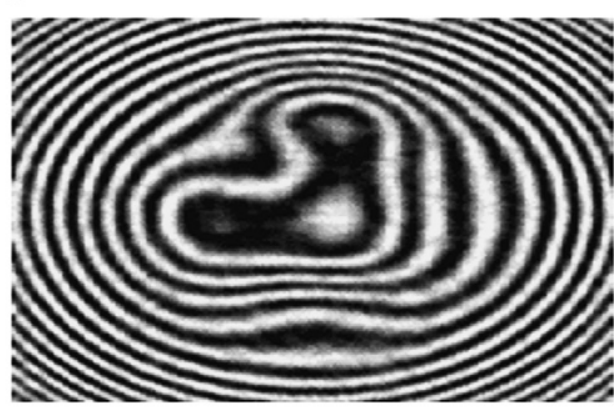

d

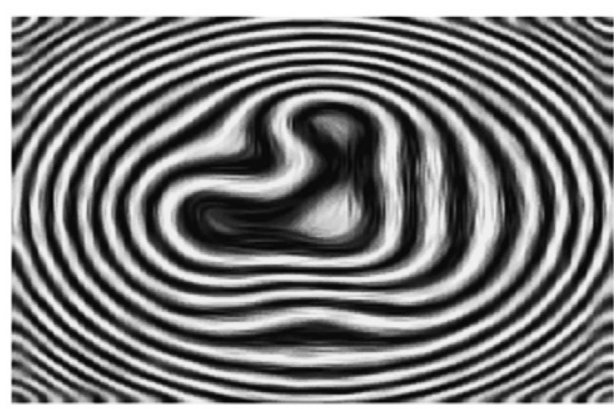

$f$

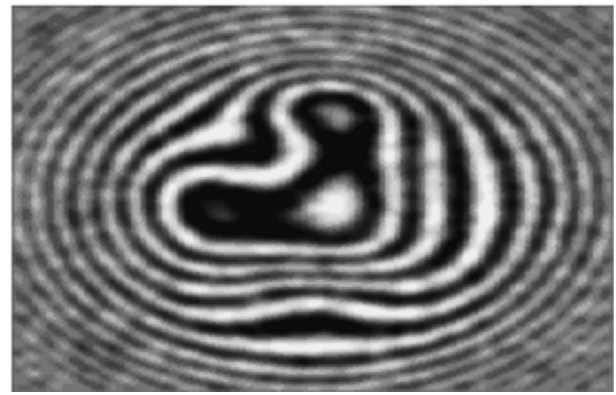

Fig. 2. Simulated Fringe pattern (a), denoised pattern by the proposed method (b), by the Wang (c) and by the Tang method (d). 
standard deviation of a Gaussian kernel which is convolved with the image before the gradient is calculated, and with the elements of the structure tensor before the diffusion tensor is obtained correspond to 3 and 10, respectively. Finally, the step-size, which establishes how much the image is modified in each iteration and the total number of iterations used are 0.1 and 200 iterations. Note that our implementation of the Wang and Tang methods requires six and five parameters respectively. In our implementation of the Wang method, we compute the fringe density map using the method proposed in [11] that has given better results than the one suggested in [4]. The obtained root-mean-square ( $r m s)$ errors between the denoised and the simulated fringe pattern without noise are $0.17,0.20,0.21,0.37$ and 0.44 (a.u) when processing with the DR, the Wang and Tang methods and with the convolutionbased denoising filter using 1 and 20 iterations. From Fig. 2(f) we see that the denoising convolution based method causes a considerable lost in fringe contrast when is used in an iterative way using 20 iterations. The processing times using a $2.67 \mathrm{GHz}$ laptop and MATLAB are given in Table 1 . In this simulation, the proposed method shows the highest accuracy in terms of rms error between the denoised and the simulated fringe pattern without noise, and presents less image artifacts than the Wang and Tang methods. Additionally, the dimensionality reduction method is approximately

Table 1

Obtained processing times by the proposed method, Wang and Tang approaches and using the convolution based methods after one and 20 iterations.

\begin{tabular}{lcllll}
\hline & DR $(\mathrm{s})$ & Wang $(\mathrm{s})$ & Tang $(\mathrm{s})$ & Conv-1 (s) & Conv-20 (s) \\
\hline Fig. 2(a) & 9.3 & 34 & 30 & $1 \times 10^{-3}$ & $1 \times 10^{-3}$ \\
Fig. 4(a) & 17 & 68 & 58 & $5 \times 10^{-3}$ & $5 \times 10^{-3}$ \\
Fig. 5(a) & 18 & 61 & 57 & $7 \times 10^{-3}$ & $7 \times 10^{-3}$ \\
\hline
\end{tabular}

three times faster than the Wang and Tang methods. In order to quantify the effect of the image artefacts and possible bluring, we have obtained the modulating phases from the denoised images shown in Fig. 2 and using the method presented in [12]. We have compared the obtained results with the ground-truth modulating phase in terms of rms errors. In Fig. 3, we show the ground-truth (a) and the reconstructed wrapped phases (b-d). Note that we have not shown the modulating phases obtained from the denoised images using the convolution based method because it is not possible to obtain the phase reconstructions from these patterns using the method presented in [10]. The obtained rms errors by the proposed, the Wang and Tang methods are $0.23,0.31$ and 0.65 (rad), respectively. Observe that the proposed method has obtained the reconstructed phase with the highest accuracy and considerably faster than the methods presented in [3,4]. Additionally, our implementation of the methods $[3,4]$ requires providing 6 userdefined parameters while our dimensionality reduction method only requires three user-defined parameters. In order to study the dependence of the proposed algorithm with respect to $n$ and $K$ parameters, we have obtained the rms errors between the groundtruth modulating phase and the phases computed from the denoised patterns with different parameters of $n$ and $K$. In Fig. 4(a) we show the results obtained when $n=25$ and we vary the number of rotations $(K)$. As can be seen from Fig. 4(a), in this case there is not a significant increase in the accuracy of the reconstructed phase when we perform a number of rotations larger than 15. In Fig. 4(b) we study the dependence of the obtained accuracy ( $r m s$ ) with respect to the number of largest eigenvalues that are not truncated $(n)$. In this case, $K$ is fixed to 15 . As can be seen from Fig. 4(b), the accuracy is low for small and large values of $n$. Note that for small values of $n$ the dimensionality reduction performed is too high and we are filtering both the noise and the signal. On the other hand, if $n$ is large the dimensionality reduction is low and the noise is not efficiently subtracted.

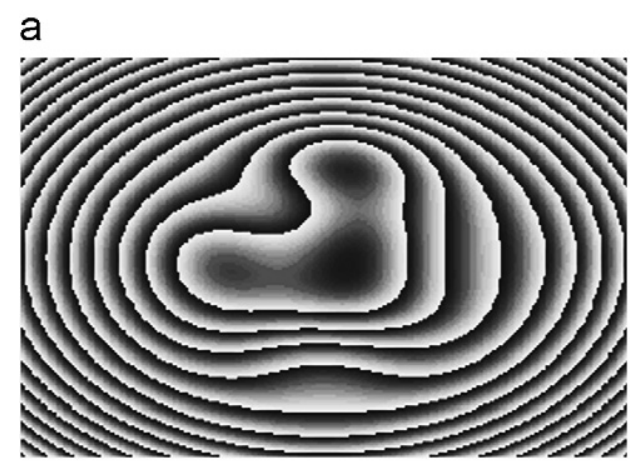

b
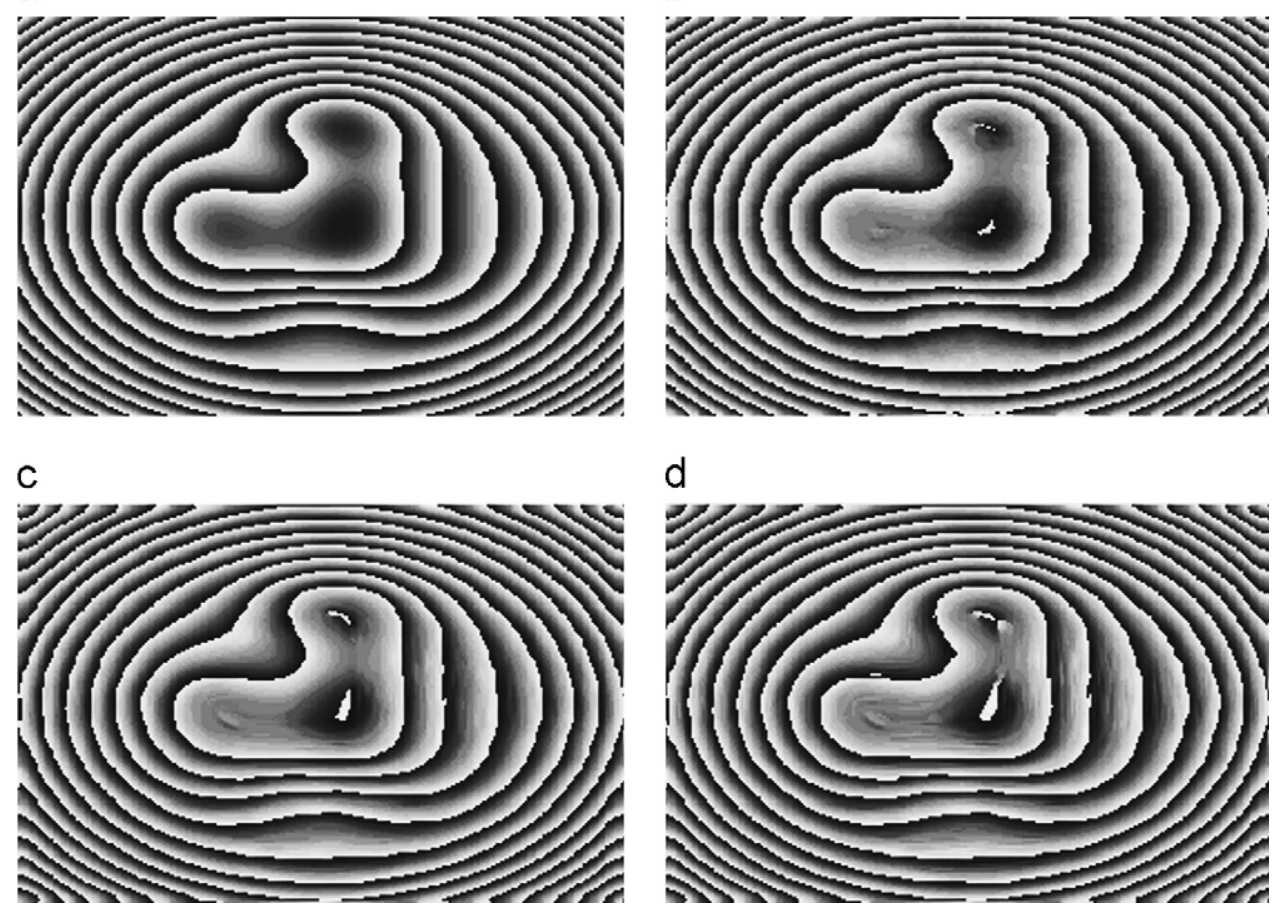

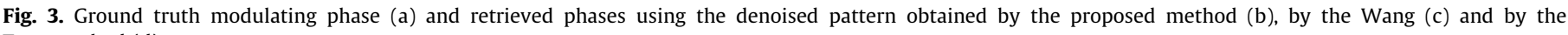
Tang method (d). 


\section{Experimental results}

We have also tested the proposed method with real ESPI images. The size of the images is $512 \times 512$. The two real ESPI patterns are obtained using double-exposure out-of-plane vibration measurements

a

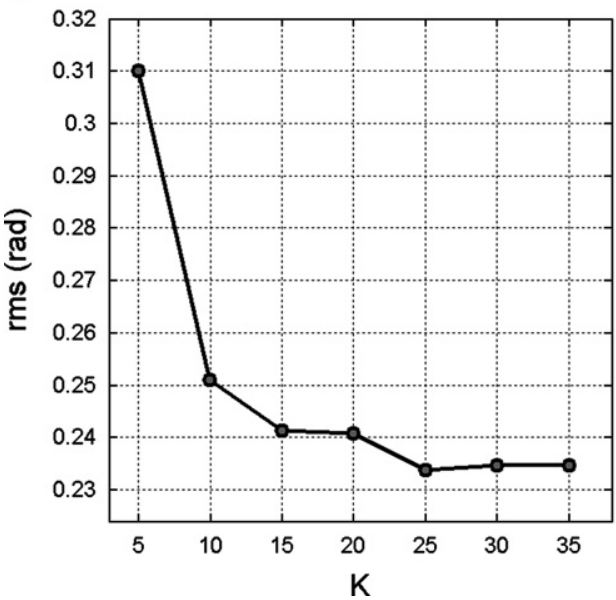

of two different vibrating surfaces. The first case, which is shown in Fig. 5(a), was taken using an ultra fast camera at 2500 frame per second, while the second one and shown in Fig. 6(a) was acquired using a conventional camera. In Figs. 5 and 6 we show two different ESPI images and the resultant denoised images obtained using the

b

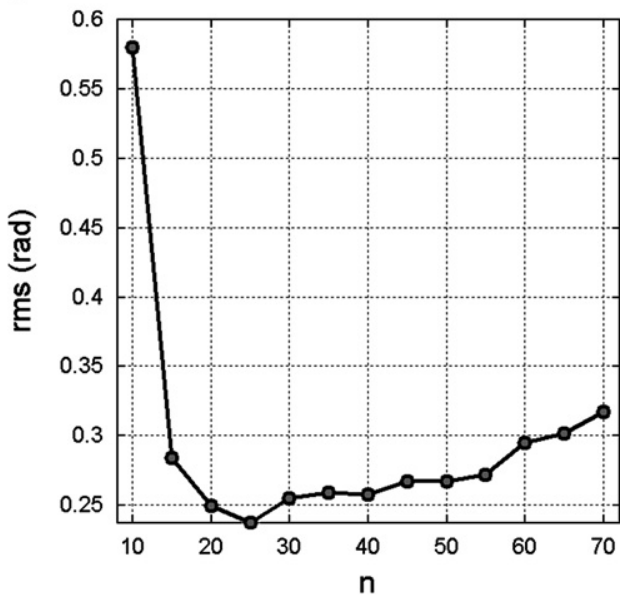

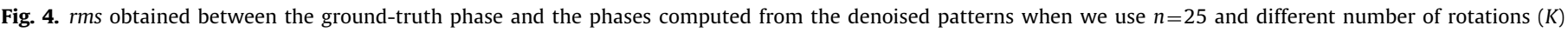
(a) and $K=15$ with different values of $n$ (b).

a

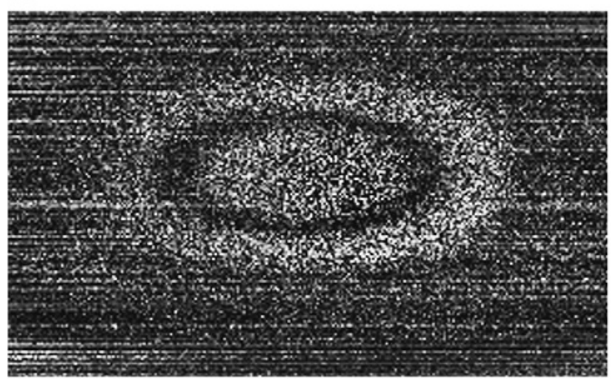

C

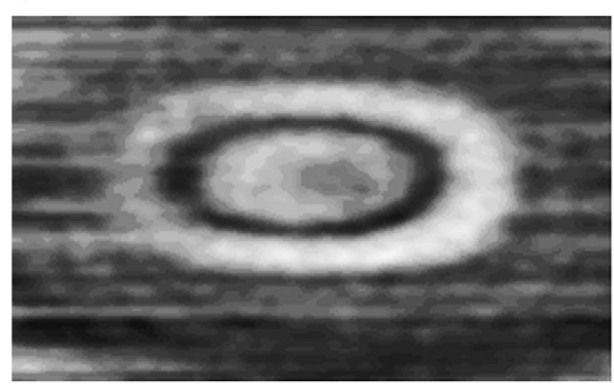

e

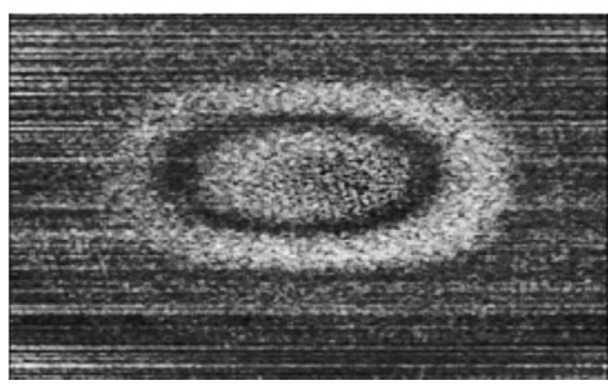

b

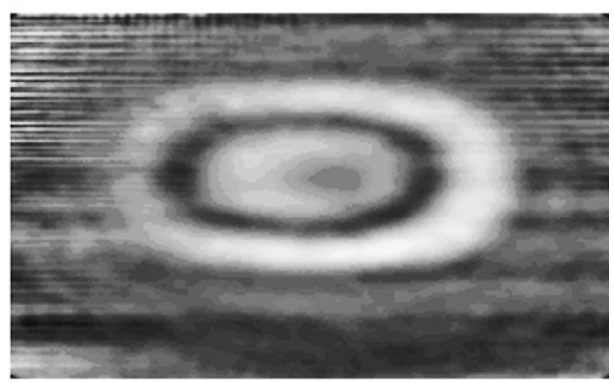

d

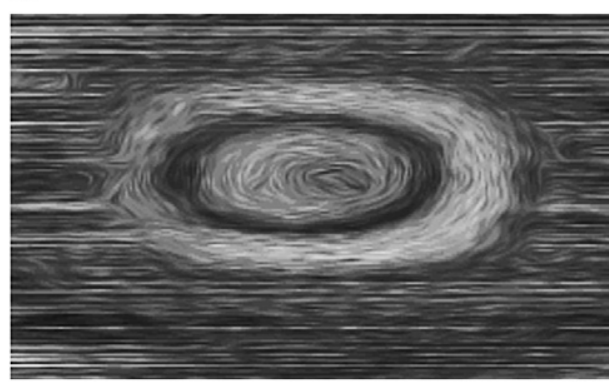

f

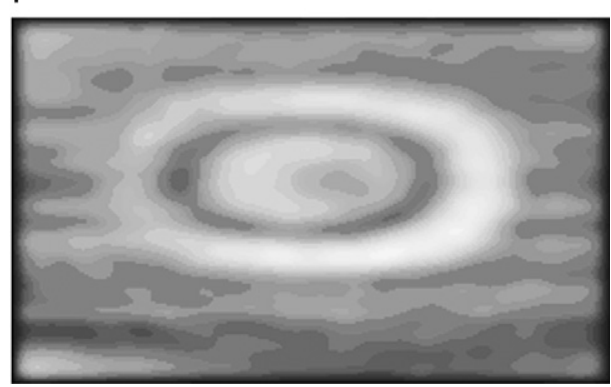

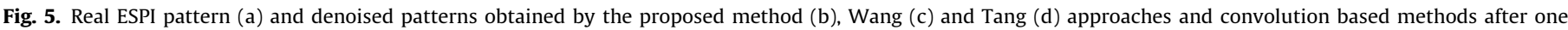
(d) and 20 (e) iterations using in both cases an average kernel of $5 \times 5$. 
a

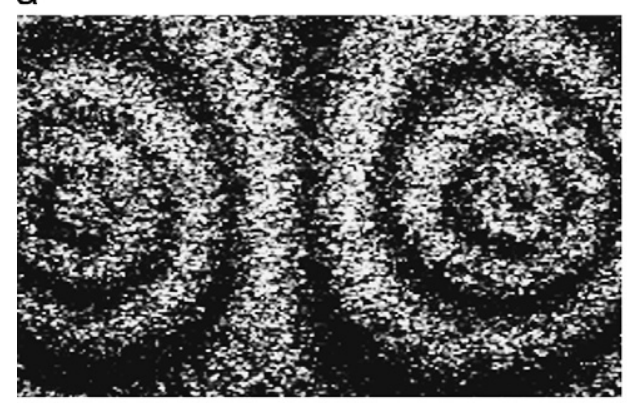

C

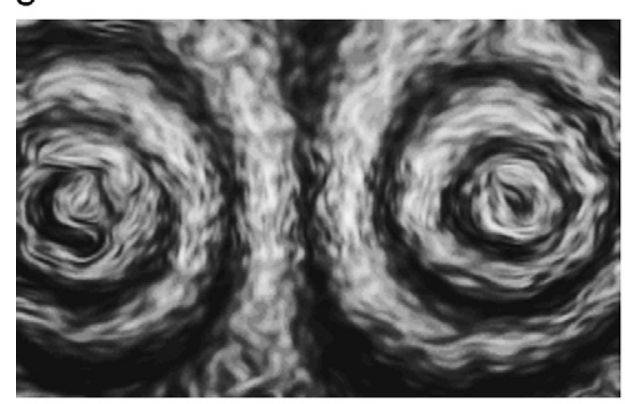

e

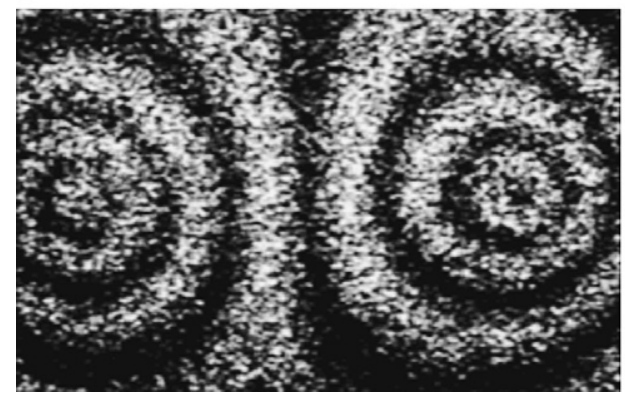

b

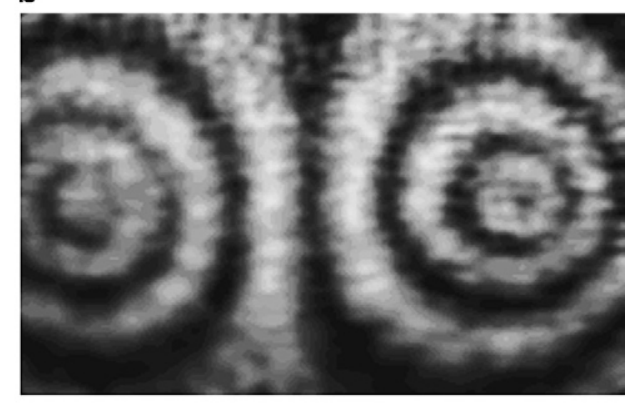

d

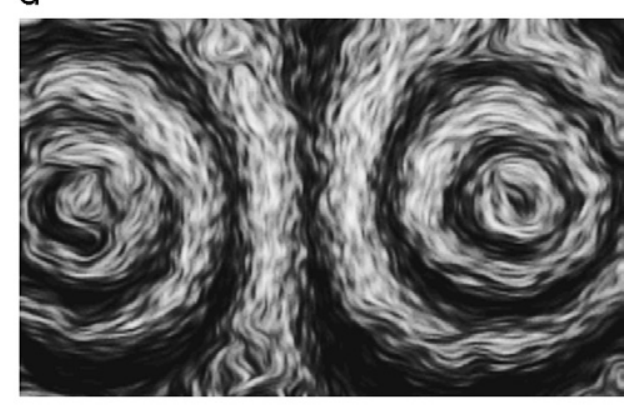

f

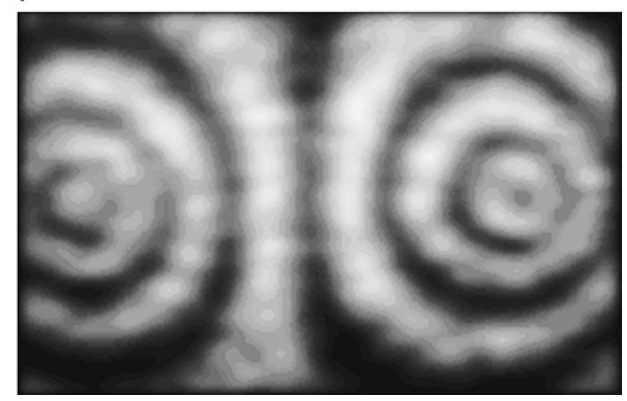

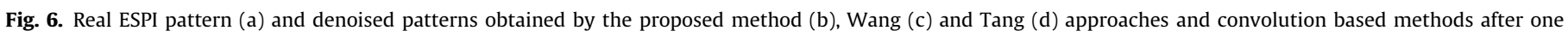
(d) and 20 (e) iterations using in both cases an average kernel of $5 \times 5$.

proposed, Wang and Tang methods and by convolution based approaches using an average kernel of size $5 \times 5$ and after 1 and 20 iterations respectively. We use 15 rotations $(K=15)$, a rank truncation of $25(n=25)$ and two iterations as input arguments of the DR method in both cases. The results obtained by the Tang and Wang methods have been acquired using the same parameters than in the case presented before. We give the different processing times in Table 1. As can be seen from Table 1, the proposed method is approximately three times faster than the Wang and Tang methods and introduces less image artifacts that these methods preserving better the local features without an appreciable lost of fringe contrast. Additionally, from Figs. 4 and 5 we see that a denoising convolution based method is ineffective when it is used after one iteration to process an ESPI image. On the other hand, this method causes a considerable lost in fringe contrast when is used in an iterative way using twenty iterations. Note for example that in Fig. 5(f) we cannot see the horizontal scanning lines of the camera that can be clearly observed in Fig. 5(b), (c) and (d).

\section{Conclusions}

In conclusion, we have proposed a straightforward denoising method, fast and easy to implement based on a dimensionality reduction method that uses the Singular Value Decomposition algorithm (SVD). The proposed denoising method only requires three user-defined input parameters. Note that the different fringe patterns shown in this work has been processed by the proposed method using the same input parameters that are $K=15, n=25$ and two iterations. This approach does not require a fine tuning of the input parameters so it is easy to obtain reliable measures. We have applied the proposed method to simulated and real ESPI patterns and we have compared the results with the results obtained by Tang [3] and Wang [4] methods, obtaining satisfactory results.

\section{Acknowledgement}

The authors would like to acknowledge economical support from the Spanish Ministry of Economy and Competitiveness through Grants ACI2009-1022, ACI2010-108 and BIO2010-16566, the Spanish Ministry of Science and Technology under Grant DPI2009-09023, and postdoctoral "Juan de la Cierva" grant with reference JCI-2011-10185. C.O.S. Sorzano is recipient of a Ramón y Cajal fellowship.

\section{References}

[1] Kaufmann GH, editor. Advances in speckle metrology and related techniques. Wiley-VCH; 2011 〈http://www.amazon.com/Advances-Speckle-MetrologyRelated-Techniques/dp/3527409572 $>$. 
[2] Perona P, Malik J. Scale-space and edge detection using anisotropic difusión. IEEE Trans Pattern Anal Mach Intell 1990;12:629.

[3] Tang C, Han L, Ren H, Zhou D, Chang Y, Wang X, et al. Second-order oriented partial-differential equations for denoising in electronic-speckle-pattern interferometry fringes. Opt Lett 2008;33:2179.

[4] Wang H, Kemao Q, Gao W, Lin F, Seah S. Fringe pattern denoising using coherence enhancing diffusion. Opt Lett 2009;34:1141.

[5] <http://www.mathworks.es/matlabcentral/fileexchange/3710-nonlinear-dif fusion- toolbox/content/cedif.m $\rangle$.

[6] Wu P, Manjunath BS, Shin HD. Dimensionality reduction for image retrieval. Proc ICPI 2000;3.

[7] Fodor IK. A survey of dimension reduction techniques. Technical report UCRLID-148494; 2002.
[8] Verbeek J. Learning nonlinear image manifolds by global alignment of local linear models. IEEE Trans Pattern Anal Mach Intell 2006;28.

[9] Kambhatla N, Leen TK. Dimension reduction by local principal component analysis. Neural Comp 1997;9(7).

[10] Carreira-Perpinan MA. A review of dimension reduction techniques. Technical report CS-96-09; 1997.

[11] Vargas J, Antonio Quiroga J, Belenguer T. Local fringe density determination by adaptive filtering. Opt Lett 2011:36:70-2.

[12] Villa J, De la Rosa I, Miramontes G, Quiroga JAntonio. Phase recovery from a single fringe pattern using an orientational vector-field-regularized estimator. J Opt Soc Am A 2005;22:2766-73. 